\title{
Angular Dynamics of a Small Particle in Turbulence
}

\author{
F. Candelier, ${ }^{1}$ J. Einarsson, ${ }^{2}$ and B. Mehlig ${ }^{2}$ \\ ${ }^{1}$ Aix-Marseille University-IUSTI (UMR CNRS 7343), 13453 Marseille Cedex, France \\ ${ }^{2}$ Department of Physics, Gothenburg University, SE-41296 Gothenburg, Sweden \\ (Received 8 June 2016; revised manuscript received 24 September 2016; published 10 November 2016)

\begin{abstract}
We compute the angular dynamics of a neutrally buoyant nearly spherical particle immersed in an unsteady fluid. We assume that the particle is small, that its translational slip velocity is negligible, and that unsteady and convective inertia are small perturbations. We derive an approximation for the torque on the particle that determines the first inertial corrections to Jeffery's equation. These corrections arise as a consequence of local vortex stretching and can be substantial in turbulence, where local vortex stretching is strong and closely linked to the irreversibility of turbulence.
\end{abstract}

DOI: 10.1103/PhysRevLett.117.204501

The angular dynamics of small nonspherical particles in flows is often discussed in terms of Jeffery's theory [1-4], neglecting the effects of particle and fluid inertia. It is assumed that the instantaneous torque on the particle vanishes at every instant in time, so that the angular velocity of a small spherical particle equals half of the fluid vorticity at the particle position. But this is no longer true when the particle is so large that inertial effects become important. It is straightforward to take into account particle inertia [3,5-7]. This may be a good approximation for heavy particles, but for neutrally buoyant particles the acceleration of the surrounding fluid cannot be neglected. How to model the effect of fluid inertia on the angular motion of a suspended particle is an important question [4,7] yet difficult to answer. Past theoretical studies have therefore often concentrated on simple cases, for example, on the angular dynamics of axisymmetric particles in shear flows, analyzing the stability of Jeffery orbits under inertial perturbations [8-11].

In this Letter, we estimate the first inertial contributions to the angular dynamics of a nearly spherical particle in a time-dependent and spatially varying flow. Our three most important assumptions are that the particle is small, almost neutrally buoyant, and nearly spherical (details are given below). We find that a spherical particle rotates at an angular velocity different from the local flow vorticity. The difference is caused by local vortex stretching. For nearly spherical particles, we find additional contributions that depend in a more complex manner on the local fluidvelocity gradients. In turbulence, these effects can alter the angular particle dynamics substantially. Recently, the question was raised how the dynamics of tracer particles reflects the irreversibility of turbulence [12-15]. Our results show how the inertial angular dynamics of a small particles

Published by the American Physical Society under the terms of the Creative Commons Attribution 3.0 License. Further distribution of this work must maintain attribution to the author(s) and the published article's title, journal citation, and DOI. is linked to the breaking of time-reversal invariance in turbulence.

Formulation of the problem.-Consider a small, nearly neutrally buoyant, spheroidal particle (with rotational symmetry axis $\boldsymbol{n}$ ) in a fluid. We assume that the particle is nearly spherical, and its aspect ratio $\lambda$ is close to unity: $\lambda \equiv 1+\epsilon$, where $\epsilon$ is a small parameter determining the eccentricity of the particle $-\epsilon>0$ for prolate particles, while $\epsilon<0$ for oblate particles. We assume that the particle is small: $\kappa \equiv 2 a / \ell \ll 1$. Here $2 a$ is the length of the symmetry axis of the particle, and $\ell$ is the length over which the flow can be linearized near the particle.

We assume that inertial effects matter but that they are weak. Convective inertia due to the fluid-velocity gradients is characterized by the shear Reynolds number $\mathrm{Re}_{s} \equiv$ $a^{2} s / \nu$, where $\nu$ is the kinematic viscosity of the fluid and $s$ measures the magnitudes of the fluid-velocity gradients, the strain rate. The effect of unsteady inertia depends on the time scale $\tau_{c}$ that describes how fast the boundary conditions change. The ratio of the magnitude of unsteady and convective inertia defines the Strouhal number $\mathrm{Sl} \equiv\left(s \tau_{c}\right)^{-1}$. The effect of particle inertia on the angular dynamics is determined by the Stokes number, the ratio of the rate of change of angular momentum and the torque: $\mathrm{St} \equiv\left(\rho_{p} a^{5} s\right) /\left(\tau_{c} \mu s a^{3}\right)=\left(\rho_{p} / \rho_{f}\right) \operatorname{Re}_{s}$ Sl. Here $\rho_{f}$ and $\rho_{p}$ are the mass densities of the fluid and the particle, respectively, and $\mu=\rho_{f} \nu$ is the dynamic viscosity.

We treat the effect of inertia perturbatively; this requires $\operatorname{Re}_{s}$ and $\operatorname{Re}_{s} \mathrm{Sl}$ to be small (but not too small; see below). We disregard the effect of translational slip. This is justified for small particle Reynolds number $\operatorname{Re}_{p} \equiv a v_{s} / \nu \ll \operatorname{Re}_{s}^{1 / 2}$. Here $v_{s}$ is the slip velocity. To ensure that it is small enough, we assume that the particle is approximately neutrally buoyant.

Equations of motion.-The equations that govern the angular particle dynamics read

$$
\frac{d \boldsymbol{n}}{d t}=\boldsymbol{\omega} \wedge \boldsymbol{n}, \quad\left(\square \cdot \frac{d \boldsymbol{\omega}}{d t}+\frac{d \llbracket}{d t} \cdot \boldsymbol{\omega}\right)=\boldsymbol{T} .
$$


Here $\llbracket$ is the moment-of-inertia tensor of the particle, with elements $I_{i j}=A^{I} n_{i} n_{j}+B^{I}\left(\delta_{i j}-n_{i} n_{j}\right), A^{I}$ and $B^{I}$ are the moments of inertia around and transverse to the axis $\boldsymbol{n}$, and $\boldsymbol{T}$ is the hydrodynamic torque:

$$
\boldsymbol{T}=\int_{S_{p}} \boldsymbol{r} \wedge \boldsymbol{\sigma} \cdot d \boldsymbol{s}
$$

The integral is over the particle surface $S_{p}, d s$ is the outward normal surface element, $\boldsymbol{\sigma}(\boldsymbol{x}, t)$ is the stress tensor of the fluid at position $\boldsymbol{x}$, and $\boldsymbol{r} \equiv \boldsymbol{x}-\boldsymbol{x}_{p}$, where $\boldsymbol{x}_{p}$ is the particle position. The torque (2) is determined by the solution of Navier-Stokes equations. We decompose the stress as $\boldsymbol{\sigma}=\boldsymbol{\sigma}^{\infty}+\boldsymbol{\sigma}^{(1)}$, where $\boldsymbol{\sigma}^{\infty}$ is the stress tensor of the undisturbed Eulerian fluid velocity, denoted by $\boldsymbol{U}^{\infty}(\boldsymbol{x}, t)$. The second term, $\boldsymbol{\sigma}^{(1)}$, is the contribution to the stress tensor from the disturbance flow. The torque is decomposed in a similar way: $\boldsymbol{T}=\boldsymbol{T}^{\infty}+\boldsymbol{T}^{(1)}$. We compute these two contributions to the torque separately.

Torque due to stress of undisturbed flow.-The undisturbed Eulerian fluid velocity $\boldsymbol{U}^{\infty}(\boldsymbol{x}, t)$ satisfies NavierStokes equations in the laboratory frame:

$$
\boldsymbol{\nabla} \cdot \boldsymbol{\sigma}^{\infty}=\rho_{f}\left[\left(\partial_{t} \boldsymbol{U}^{\infty}\right)_{\boldsymbol{x}}+\left(\boldsymbol{U}^{\infty} \cdot \boldsymbol{\nabla}\right) \boldsymbol{U}^{\infty}\right] \equiv \rho_{f} \frac{D}{D t} \boldsymbol{U}^{\infty} .
$$

The last equality defines the Lagrangian derivative along $\boldsymbol{U}^{\infty}, \boldsymbol{\nabla}$ denotes the spatial derivative with respect to $\boldsymbol{x}$, and the partial time derivative is evaluated at fixed $\boldsymbol{x}$. The torque due to the undisturbed stress can be expressed as a volume integral using Eq. (3):

$$
\boldsymbol{T}^{\infty}=\rho_{f} \int_{V_{p}} d v \boldsymbol{r} \wedge \frac{D \boldsymbol{U}^{\infty}}{D t} .
$$

To evaluate this expression further, we expand $D \boldsymbol{U}^{\infty}(\boldsymbol{x}, t) / D t$ around the particle position $\boldsymbol{x}_{p}$ :

$$
\begin{aligned}
\left.\frac{D U_{k}^{\infty}}{D t}\right|_{\boldsymbol{x}}= & \left.\frac{D U_{k}^{\infty}}{D t}\right|_{\boldsymbol{x}_{p}}+\left.r_{m} \frac{\partial}{\partial x_{m}} \frac{D U_{k}^{\infty}}{D t}\right|_{\boldsymbol{x}_{p}} \\
& +\left.r_{m} r_{n} \frac{\partial}{\partial x_{m}} \frac{\partial}{\partial x_{n}} \frac{D U_{k}^{\infty}}{D t}\right|_{\boldsymbol{x}_{p}}+O\left(r^{3} / \ell^{3}\right) .
\end{aligned}
$$

The components are relative to a fixed Cartesian basis in the laboratory frame. Substituting this expansion into Eq. (4), we find

$T_{i}^{\infty}=\rho_{f} \int_{V_{p}} d^{3} r \varepsilon_{i j k} r_{j}\left(\left.\frac{\partial}{\partial x_{m}} \frac{D U_{k}^{\infty}}{D t}\right|_{\boldsymbol{x}_{p}}\right) r_{m}+O\left(r^{4} / \ell^{4}\right)$.

The order is $O\left(r^{4} / \ell^{4}\right)$, because terms odd in $\boldsymbol{r}$ vanish upon integration. The partial derivative in the integrand of Eq. (6) evaluates to $\left.\boldsymbol{\nabla}\left(D \boldsymbol{U}^{\infty} / D t\right)\right|_{x_{p}}=\left.\left(D \mathbb{A}^{\infty} / D t\right)\right|_{x_{p}}+\mathbb{A}_{p}^{\infty} \cdot \mathbb{A}_{p}^{\infty}$, where the elements of $\mathbb{A}_{p}^{\infty}$ are the gradients of the undisturbed fluid velocity $\boldsymbol{U}^{\infty}$ at the particle center: $\left(\mathbb{A}_{p}^{\infty}\right)_{i j} \equiv \partial U_{i}^{\infty} /\left.\partial x_{j}\right|_{x=x_{p}}$.

Finally, to perform the integral in Eq. (6) we use the definition of the moment-of-inertia tensor. We obtain

$$
\begin{aligned}
\boldsymbol{T}^{\infty} & =\frac{\rho_{f}}{\rho_{p}}\left\{\square \cdot\left(\left.\frac{D \Omega^{\infty}}{D t}\right|_{\boldsymbol{x}_{p}}-\mathbb{S}_{p}^{\infty} \cdot \Omega_{p}^{\infty}\right)\right. \\
& \left.+\left(A^{I}-B^{I}\right)\left[\left(\mathbb{S}_{p}^{\infty} \cdot \mathbb{S}_{p}^{\infty}+\mathbb{O}_{p}^{\infty} \cdot \mathbb{O}_{p}^{\infty}+\left.\frac{D \mathbb{S}^{\infty}}{D t}\right|_{\boldsymbol{x}_{p}}\right) \cdot \boldsymbol{n}\right] \wedge \boldsymbol{n}\right\} .
\end{aligned}
$$

Here we have decomposed the matrix $\mathbb{A}_{p}^{\infty}$ into its symmetric and antisymmetric parts, the strain-rate matrix $\mathbb{S}_{p}^{\infty}$, and $\mathbb{O}_{p}^{\infty}$. The matrix $\mathbb{O}_{p}^{\infty}$ is linked to $\Omega_{p}^{\infty} \equiv \frac{1}{2} \boldsymbol{\nabla} \wedge \boldsymbol{U}^{\infty}$ through $\mathbb{O}^{\infty} \cdot \boldsymbol{r}=\Omega^{\infty} \wedge \boldsymbol{r}$. Equation (7) is valid for a spheroid with an arbitrary aspect ratio (provided that $\kappa \ll 1)$.

Using the vorticity equation $\left(D \Omega^{\infty} / D t\right)-\mathbb{S}^{\infty} \cdot \Omega^{\infty}=$ $\nu \nabla^{2} \Omega^{\infty}$ evaluated at the particle position, we can express the difference in the first row of Eq. (7) in terms of the Laplacian of $\Omega^{\infty}$ which vanishes for a strictly linear flow. In general, however, the nonlinearity of $\boldsymbol{U}^{\infty}(\boldsymbol{x}, t)$ results in a nonzero value of $\nabla^{2} \Omega^{\infty}$ at the particle position.

Disturbance torque.-We calculate the torque due to the disturbed fluid in the perturbation theory, assuming that inertial effects are small and neglecting translational slip. This dictates how the problem must be dedimensionalized: $t=\tau_{c} t^{\prime}, \boldsymbol{r}=a \boldsymbol{r}^{\prime}, \boldsymbol{U}=s a \boldsymbol{U}^{\prime}$, and $\boldsymbol{\sigma}=\mu s \boldsymbol{\sigma}^{\prime}$. In the remainder of this Letter, we use these dimensionless variables. To simplify the notation, we drop the primes; all equations below are written in dimensionless variables. The disturbance caused by the particle has the flow velocity $\boldsymbol{w}(\boldsymbol{r}, t) \equiv \boldsymbol{U}\left(\boldsymbol{r}+\boldsymbol{x}_{p}, t\right)-\boldsymbol{U}^{\infty}\left(\boldsymbol{r}+\boldsymbol{x}_{p}, t\right)$. It is defined to be a function of $\boldsymbol{r}(t) \equiv \boldsymbol{x}-\boldsymbol{x}_{p}(t)$ (Fig. 1). In dimensionless variables, the disturbance problem reads

$$
\begin{aligned}
& \nabla_{\boldsymbol{r}} \cdot \boldsymbol{\sigma}^{(1)}= \operatorname{Re}_{s}\left[\operatorname{Sl}\left(\partial_{t} \boldsymbol{w}\right)_{\boldsymbol{r}}+\left(\mathbb{A}_{p}^{\infty} \cdot \boldsymbol{r}\right) \cdot \nabla_{\boldsymbol{r}} \boldsymbol{w}+\mathbb{A}_{p}^{\infty} \cdot \boldsymbol{w}\right. \\
&\left.+\left(\boldsymbol{w} \cdot \nabla_{\boldsymbol{r}}\right) \boldsymbol{w}\right], \\
& \boldsymbol{w}(\boldsymbol{r}, t)=-\left(\mathbb{A}_{p}^{\infty} \cdot \boldsymbol{r}-\boldsymbol{\omega} \wedge \boldsymbol{r}\right) \quad \text { for } \boldsymbol{r} \in S_{p}, \\
& \boldsymbol{w}(\boldsymbol{r}, t)=0 \quad \text { as } \quad|\boldsymbol{r}| \rightarrow \infty .
\end{aligned}
$$

The partial time derivative is evaluated at fixed $\boldsymbol{r}$, and we have linearized $\boldsymbol{U}^{\infty}(\boldsymbol{x}, t)$ around $\boldsymbol{x}_{p}, \boldsymbol{U}^{\infty}(\boldsymbol{x}, t)=$ $\boldsymbol{U}^{\infty}\left(\boldsymbol{x}_{p}, t\right)+\mathrm{A}_{p}^{\infty} \cdot \boldsymbol{r}$. When is it justified to use this linear form in the disturbance problem (8)? The disturbance caused by the particle decays exponentially at distances larger than the Saffman length $\ell_{S} \equiv 1 / \sqrt{\operatorname{Re}_{s}}$, so that we must require $\ell_{S}<\ell$, where $\ell$ is the length scale over which the flow can be linearized. This condition is more restrictive than $\kappa \ll 1$. In other words, the shear Reynolds number $\operatorname{Re}_{s}$ 


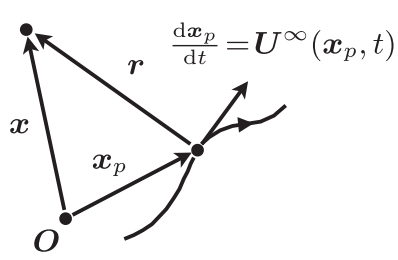

Eulerian fluid

velocity $\boldsymbol{U}(\boldsymbol{x}, t)$

Undisturbed Eulerian

fluid velocity $\boldsymbol{U}^{\infty}(\boldsymbol{x}, t)$

Disturbance flow

$\boldsymbol{w}(\boldsymbol{r}, t)=\boldsymbol{U}\left(\boldsymbol{r}+\boldsymbol{x}_{p}, t\right)$

$$
-\boldsymbol{U}^{\infty}\left(\boldsymbol{r}+\boldsymbol{x}_{p}, t\right)
$$

FIG. 1. Schematic of vectors used to describe the particle motion. $\boldsymbol{x}$ is a position in the laboratory frame, $\boldsymbol{O}$ is its origin, and $\boldsymbol{x}_{p}(t)$ is the particle position at time $t$. The perturbation theory leading to Eq. (13) assumes that the particle follows the Lagrangian fluid trajectory.

should not be too small, because convective inertia causes the disturbance to decay at $\ell_{s}$.

We use the reciprocal theorem [16] to find the hydrodynamic torque on the particle, given an "auxiliary" Stokes solution in the same geometry $[9,10,17,18]$. In dimensionless variables, the reciprocal theorem reads

$$
\boldsymbol{T}=\boldsymbol{T}^{\infty}+\boldsymbol{T}^{(0)}-\operatorname{Re}_{s} \int_{V} d v \mathbb{M}^{\top} \cdot \boldsymbol{f}(\boldsymbol{w}) .
$$

The first term on the right-hand side is the torque due to the undisturbed fluid stresses, Eq. (7), expressed in dimensionless variables. The second term is Jeffery's torque [1]: $\boldsymbol{T}^{(0)}=-\mathbb{K} \cdot\left(\boldsymbol{\omega}-\Omega_{p}^{\infty}\right)+\mathbb{U}: \mathbb{S}_{p}^{\infty}$, where $\mathbb{K}$ and $\mathbb{\square}$ are Brenner's resistance tensors [19]. The third term in Eq. (9) is the torque due to the disturbance flow beyond the Stokes approximation. The tensor $\mathbb{M}(\boldsymbol{r})$ is determined by the known auxiliary Stokes solution $(\tilde{\boldsymbol{u}}=\mathbb{M} \cdot \tilde{\boldsymbol{\omega}})$, the Stokes flow around the particle rotating with angular velocity $\tilde{\boldsymbol{\omega}}$ in a quiescent fluid; see Supplemental Material [20]. The integration domain in (9) is the fluid volume outside the particle, and $\operatorname{Re}_{s} f$ is defined as the right-hand side of the first Eq. (8).

To simplify the calculation of the third term in Eq. (9), we assume that the particle is nearly spherical [18], $\lambda=1+\epsilon$, and expand in the small parameter $\epsilon$. To the order of $\epsilon^{2}$, for example, the moments of inertia around and transverse to the particle symmetry axis [18] read in dimensionless variables $A^{I}=(8 \pi / 15)\left(1-4 \epsilon+6 \epsilon^{2}\right)$ and $B^{I}=(8 \pi / 15)\left(1-3 \epsilon+(7 / 2) \epsilon^{2}\right)$. For small values of $\epsilon$. the contribution of the volume integral in Eq. (9) can be evaluated. Details are given in Supplemental Material [20].

For a spherical particle $(\epsilon=0)$, we find to the order of $O\left(\operatorname{Re}_{s}\right)$

$\boldsymbol{T}=-8 \pi\left(\boldsymbol{\omega}-\Omega_{p}^{\infty}\right)+8 \pi \operatorname{Re}_{s}\left(\left.\frac{\mathrm{S} 1}{15} \frac{D \Omega^{\infty}}{D t}\right|_{x_{p}}-\frac{2}{5} \mathbb{S}_{p}^{\infty} \cdot \Omega_{p}^{\infty}\right)$.

We now use Eqs. (10) and (1) to determine the particle angular velocity $\omega$ to the order of $O\left(\operatorname{Re}_{s}\right)$. For a spherical particle, $\square$ has the elements $I_{i j}=8 \pi \delta_{i j} / 15$, in dimensionless variables. In the perturbation theory, $d \omega / d t=d \Omega_{p}^{\infty} / d t=$ $D \Omega^{\infty} /\left.D t\right|_{x_{p}}$. We must also require that $\rho_{p} \approx \rho_{f}$, so that $\operatorname{Re}_{p}$ remains small. To the order of $O\left(\operatorname{Re}_{s}\right)$ we find

$\omega=\Omega_{p}^{\infty}+\left.\frac{1}{15}\left(\operatorname{Re}_{s} \mathrm{Sl}-\mathrm{St}\right) \frac{D \Omega^{\infty}}{D t}\right|_{x_{p}}-\frac{2}{5} \operatorname{Re}_{s} \mathbb{S}_{p}^{\infty} \cdot \Omega_{p}^{\infty}$.

Equation (11) is the main result of this Letter. We see that a very small particle in a viscous flow rotates with half the fluid vorticity, $\Omega_{p}^{\infty}$, as expected. The first inertial correction term, proportional to $D \Omega^{\infty} /\left.D t\right|_{x_{p}}$, resembles the form of the slip velocity of a small particle subject to particle inertia and to the force due to the undisturbed pressure gradients. A small-St expansion gives

$$
\boldsymbol{v}_{s}=\operatorname{St}\left(\rho_{f} / \rho_{p}-1\right) D \boldsymbol{U}^{\infty} /\left.D t\right|_{x_{p}}
$$

for $\mathrm{Sl}=1$. Maxey used this approximation to conclude that heavy particles are centrifuged out of vortical regions in turbulence [22,23]. The expression for $\boldsymbol{v}_{s}$ is of the same form as the second term on the right-hand side of Eq. (11), since $\operatorname{Re}_{s}=\left(\rho_{f} / \rho_{p}\right) \mathrm{St}$ for $\mathrm{Sl}=1$. This term predicts that a particle that is slightly heavier than the fluid rotates a little bit more slowly, because it cannot keep up with the fluid acceleration. A lighter particle, by contrast, rotates faster than $\Omega_{p}^{\infty}$. But these arguments disregard the $\mathbb{S}_{p}^{\infty} \cdot \Omega_{p}^{\infty}$-term in Eq. (11). This inertial term connects the angular particle dynamics to vortex stretching in the undisturbed flow. This term vanishes in steady linear flows such as a simple shear $[24,25]$ and for planar flows because vorticity is orthogonal to the flow plane.

Angular velocity and vortex stretching in turbulence.Fully developed turbulent flows exhibit large local vortex stretching rates; this is how the turbulent kinetic energy is dissipated at small scales. Irreversibility of turbulence [12-15] implies that the stretching rate $\Omega^{\infty} \cdot\left(\mathbb{S}^{\infty} \cdot \Omega^{\infty}\right)$ does not average to zero [26,27]. This matrix element is positive on average, because $\Omega^{\infty}$ tends to align with the middle eigenvector of $\mathbb{S}^{\infty}$ [12], and its eigenvalue is positive on average. Equation (11) shows that the inertial correction to the angular velocity of a neutrally buoyant sphere in turbulence is determined by the stretching rate,

$$
|\boldsymbol{\omega}|^{2} /\left|\Omega_{p}^{\infty}\right|^{2} \approx 1-\frac{4}{5} \operatorname{Re}_{s} \Omega_{p}^{\infty} \cdot\left(\mathbb{S}_{p}^{\infty} \cdot \Omega_{p}^{\infty}\right) /\left|\Omega_{p}^{\infty}\right|^{2},
$$

and thus linked to the breaking of time-reversal invariance in turbulence.

But are the conditions of validity summarized above met for a neutrally buoyant particle in turbulence? The shear rate, defined by $s=\langle\operatorname{Tr} \mathbb{S} \cdot \mathbb{S}\rangle^{1 / 2}$, is of the same order as $\tau_{K}^{-1}$, the inverse of the Kolmogorov time $\tau_{K} \equiv$ $\left\langle\operatorname{Tr} \mathbb{A}^{\top} \cdot \mathbb{A}\right\rangle^{-1 / 2}$. This means that the shear Reynolds number is of the order of $\operatorname{Re}_{s} \sim a^{2} / \eta_{K}^{2}$, where $\eta_{K}=\sqrt{\nu \tau_{K}}$ is the 

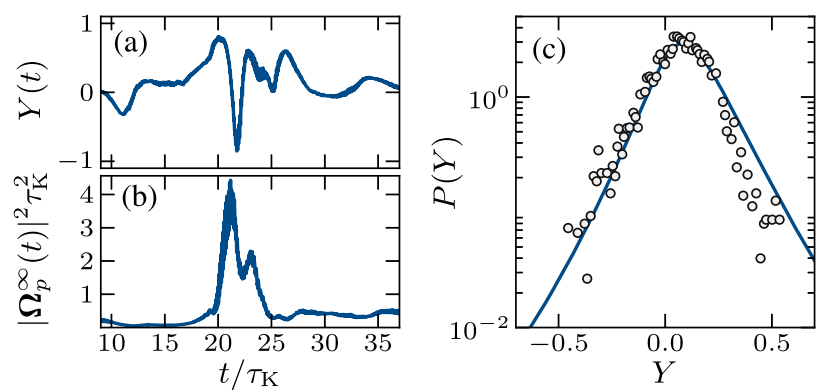

FIG. 2. (a) Inertial correction $Y \equiv \tau_{K} \Omega_{p}^{\infty} \cdot \mathbb{S}_{p}^{\infty} \cdot \Omega_{p}^{\infty} /\left|\Omega_{p}^{\infty}\right|^{2}$ [Eq. (13)] along a Lagrangian path in turbulence (DNS at $\operatorname{Re}_{\lambda}=$ 433 using the JHU turbulence database [28,29]). (b) Vorticity along the same path. (c) Distribution of $Y$ (DNS, lines). Also shown: experimental data (circles) at $\operatorname{Re}_{\lambda}=50 \mathrm{read}$ off from Fig. 2(d) in Ref. [26], rescaled by $\tau_{K} \equiv\left\langle\operatorname{Tr} \mathbb{A}^{\top} \cdot A\right\rangle^{-1 / 2}$; see the text. The experimental value $\left(0.27 \mathrm{~s}^{-1}\right)$ was taken from Ref. [30].

Kolmogorov length. We conclude that the particle must be smaller than the Kolmogorov length for the perturbation theory in $\operatorname{Re}_{s}$ to be valid. For a nearly neutrally buoyant particle we can disregard gravity, and we assume that no other external forces act on the particle. Therefore, the time scale $\tau_{c}$ is that of the fluid, and we take $\tau_{c}=s^{-1} \sim \tau_{K}$, so that the Strouhal number is unity. The perturbation theory also requires that the Saffman length $\ell_{S} \equiv a / \sqrt{\operatorname{Re}_{s}} \sim \eta_{K}$ is smaller than the length $\ell$ over which the flow can be linearized. For turbulent flow at Reynolds number $\operatorname{Re}_{\lambda} \sim 500, \ell \sim 10 \eta_{K}$, on average. So this condition is only marginally satisfied. Translational slip is negligible when the Oseen length is much larger than the Saffman length, that is $\operatorname{Re}_{p}^{2} \ll \operatorname{Re}_{s}$. For small neutrally buoyant particles in turbulence, this condition is well satisfied.

We evaluate the inertial correction in Eq. (13) for a neutrally buoyant particle numerically, following a Lagrangian trajectory in fully developed turbulence. We use the Johns Hopkins turbulence database [28,29] that contains a direct numerical simulation of forced, isotropic turbulence at $\operatorname{Re}_{\lambda}=433$. The result is shown in Fig. 2(a). Figure 2(b) shows the vorticity along the same path. We observe the stretching of a vortex tube at $t \approx 20 \tau_{K}$. We see that the inertial correction to the particle angular velocity can be substantial during vortex stretching, a factor of the order of unity times $\mathrm{Re}_{s}$. Figure 2(c) shows that the distribution of the inertial correction has non-Gaussian tails that may give rise to large values of the inertial correction. A comparison with the experimental data at $\operatorname{Re}_{\lambda}=50$ (o), from Fig. 2(d) in Ref. [26], shows that the tails are quite robust even at moderate values of $\operatorname{Re}_{\lambda}$, a consequence of the universality of dissipative-range turbulent fluctuations [31].

Nonspherical particles. - We have computed the angular velocity also for nearly spherical particles $(|\epsilon| \ll 1)$. Neglecting inertial effects, we obtain an $\epsilon$ expansion of Jeffery's equation [1]: $\boldsymbol{\omega}=\Omega_{p}^{\infty}+\left(\epsilon+\left(\epsilon^{2} / 2\right)\right)\left(\boldsymbol{n} \wedge \mathbb{S}_{p}^{\infty} \cdot \boldsymbol{n}\right)+O\left(\epsilon^{3}\right)$.
The form of the first inertial corrections to Jeffery's angular velocity is constrained by symmetries. To linear order in $\mathrm{St}$ and $\mathrm{Re}_{s}$ the corrections are quadratic in $\mathbb{S}_{p}^{\infty}$ and $\Omega_{p}^{\infty}$ and linear in time derivatives of $\mathbb{S}_{p}^{\infty}$ and $\Omega_{p}^{\infty}$. The inertial corrections to $\boldsymbol{\omega}$ must be invariant under $\boldsymbol{n} \rightarrow \boldsymbol{- n}$, and $\mathbb{S}_{p}^{\infty}$ is symmetric and traceless. Only the following terms can occur in the $O\left(\epsilon \mathrm{Re}_{s}\right)$ correction $\delta \omega \equiv$ $\epsilon \operatorname{Re}_{s} \omega^{\left(\epsilon \mathrm{Re}_{s}\right)}+\epsilon \operatorname{St} \omega^{(\epsilon \mathrm{St})}$ to Eq. (11):

$$
\begin{aligned}
\delta \boldsymbol{\omega}= & \left.\beta_{1} \frac{D \Omega^{\infty}}{D t}\right|_{\boldsymbol{x}_{p}}+\beta_{2}\left(\left.\frac{D \Omega^{\infty}}{D t}\right|_{\boldsymbol{x}_{p}} \cdot \boldsymbol{n}\right) \boldsymbol{n}+\beta_{3}\left(\frac{d \mathbb{S}_{p}^{\infty}}{d t} \cdot \boldsymbol{n}\right) \wedge \boldsymbol{n} \\
& +\beta_{4} \mathbb{S}_{p}^{\infty} \cdot \Omega_{p}^{\infty}+\beta_{5}\left[\boldsymbol{n} \cdot\left(\mathbb{S}_{p}^{\infty} \cdot \Omega_{p}^{\infty}\right)\right] \boldsymbol{n} \\
& +\beta_{6}\left[\boldsymbol{n} \cdot\left(\mathbb{S}_{p}^{\infty} \cdot \boldsymbol{n}\right)\right] \Omega_{p}^{\infty} \\
& +\beta_{7}\left(\boldsymbol{n} \cdot \Omega_{p}^{\infty}\right)\left(\Omega_{p}^{\infty} \wedge \boldsymbol{n}\right)+\beta_{8}\left(\mathbb{S}_{p}^{\infty} \cdot \mathbb{S}_{p}^{\infty} \cdot \boldsymbol{n}\right) \wedge \boldsymbol{n} \\
& +\beta_{9}\left[\mathbb{S}_{p}^{\infty} \cdot\left(\Omega_{p}^{\infty} \wedge \boldsymbol{n}\right)\right] \wedge \boldsymbol{n}+\beta_{10}\left(\boldsymbol{n} \cdot \Omega_{p}^{\infty}\right) \mathbb{S}_{p}^{\infty} \cdot \boldsymbol{n} .
\end{aligned}
$$

For $\mathrm{Sl}=1$ we find using the method described above (details in Supplemental Material [20])

$\beta_{1}=-\frac{3 \epsilon}{15}\left(\operatorname{Re}_{s}-\mathrm{St}\right), \quad \beta_{2}=-\frac{\epsilon}{15}\left(\operatorname{Re}_{s}-\mathrm{St}\right)$,

$\beta_{3}=-\frac{\epsilon}{3}\left(\operatorname{Re}_{s}-\frac{1}{5} \mathrm{St}\right), \quad \beta_{4}=\frac{733 \epsilon}{525} \operatorname{Re}_{s}$,

$\beta_{5}=-\frac{\epsilon}{15}\left(\frac{4}{35} \operatorname{Re}_{s}+\mathrm{St}\right), \quad \beta_{6}=-\frac{\epsilon}{15}\left(\frac{30}{35} \mathrm{Re}_{s}-\mathrm{St}\right)$,

$\beta_{7}=-\frac{\epsilon}{15}\left(\operatorname{Re}_{s}-\mathrm{St}\right), \quad \beta_{8}=-\frac{8 \epsilon}{21} \operatorname{Re}_{s}$,

$\beta_{9}=-\frac{\epsilon}{15}\left(4 \mathrm{Re}_{s}-\mathrm{St}\right), \quad \beta_{10}=\frac{3 \epsilon}{35} \mathrm{Re}_{s}$.

Equation (14) shows that the inertial corrections to the angular velocity of nonspherical particles depend intricately on the relative alignment of the particle symmetry axis, of the vorticity, and of the eigensystem of the strainrate matrix $[2,3,32]$. For a neutrally buoyant particle in incompressible isotropic homogeneous turbulence, we can average the correction. Since $\delta \omega$ contains a factor $\epsilon$, we obtain the $O\left(\epsilon \mathrm{Re}_{s}\right)$ result by averaging $\boldsymbol{n}$ uniformly over the unit sphere: $\left\langle n_{i} n_{j}\right\rangle=\delta_{i j} / 3$. This gives $\left\langle\Omega_{p}^{\infty} \cdot \delta \boldsymbol{\omega}\right\rangle=$ $(4 / 3) \epsilon \operatorname{Re}_{s}\left\langle\Omega_{p}^{\infty} \cdot\left(\mathbb{S}^{\infty} \cdot \Omega_{p}^{\infty}\right)\right\rangle$. On average, the form of the correction is similar to that in Eq. (11). We see that this correction is positive for slightly prolate neutrally buoyant particles but negative for oblate particles.

Conclusions. - We have computed the first inertial corrections to the angular velocity of a small, approximately neutrally buoyant particle in a space- and time-dependent flow. Our main prediction, Eq. (13), expresses the inertial correction to the angular velocity of a small sphere in terms of a matrix element that determines vortex stretching. This shows that the inertial angular dynamics of a small 
neutrally buoyant sphere in turbulence picks up that timereversal invariance is broken [12-15].

Our results demonstrate that convective and unsteady fluid inertia must be treated on an equal footing in turbulence. It is commonly argued that convective effects can be disregarded when the translational slip velocity $\boldsymbol{v}_{s}$ is negligible. But we have shown here that substantial inertial corrections may arise from convective terms due to turbulent strains. Such terms are likely to be important in the translational problem, too, so that the "Maxey-Riley" equations $[33,34]$ cannot be used to describe the translational dynamics of small spheres in turbulence.

Particle-tracking experiments [35-37] and particleresolving direct numerical simulations (DNS) required to test the predictions in this Letter have recently become possible $[38,39]$, but they are still very challenging. In order to stimulate the substantial effort required for these measurements, we now give a concrete suggestion for how Eq. (13) could be tested in either an experiment or particleresolving DNS. First, the particle should be neutrally buoyant, and its size $a$ must be smaller than the Kolmogorov length $\eta_{K}$. Small $a$ ensures that $\mathrm{Re}_{p}^{2} \ll \mathrm{Re}_{s}$, since $\operatorname{Re}_{p} \propto a^{3}$ [Eq. (12)] while $\operatorname{Re}_{s} \propto a^{2}$. Small particle size may be a difficult technical requirement. It is easier to meet for lower rate of energy dissipation, so that $\eta_{K}$ is larger. Figure 2(c) shows that the effect persists for lower $\operatorname{Re}_{\lambda}$. Second, to determine the vorticity of the undisturbed flow, one must measure and interpolate the flow near the particle [35]. Then we suggest to consider the distribution of $|\boldsymbol{\omega}|^{2} /\left|\Omega_{p}^{\infty}\right|^{2}-1$. The width of this distribution must approach zero for a perfect tracer particle. Figure 2(c) shows that the first effect of inertia is to substantially widen this distribution and to slightly shift its mean value. Finally, our results for nearly spherical particles indicate that disks may be more sensitive to inertial corrections than rods.

Can our results be generalized to cases where $\boldsymbol{v}_{s}$ is not negligible? An important case is settling [40-43]. The settling of ice crystals, for instance, is important for rain initiation in cold turbulent clouds [44]. For a spatially constant flow, the effect of nonzero $\boldsymbol{v}_{s}$ was analyzed by Lovalenti and Brady [16]. Can one use their methods to compute lift forces [45] on small particles in turbulence? This is a difficult problem, because it requires singular perturbation theory [46]. Finally, larger particles pose other problems: They sense inertial-range turbulent fluctuations [47], and wakes may affect their dynamics [48].

We thank Howard Stone for discussions and for making the preprint [49] available to us that considers a related question (the steady-state limit of the problem considered here). This work was supported by Vetenskapsrådet [Grant No. 2013-3992], Formas [Grant No. 2014-585], and by the grant "Bottlenecks for particle growth in turbulent aerosols" from the Knut and Alice Wallenberg
Foundation, Dnr. KAW 2014.0048. The numerical results in Fig. 2 use data from the Johns Hopkins turbulence database $[28,29]$.

[1] G. B. Jeffery, The motion of ellipsoidal particles immersed in a viscous fluid, Proc. R. Soc. A 102, 161 (1922).

[2] S. Parsa, E. Calzavarini, F. Toschi, and G. A. Voth, Rotation Rate of Rods in Turbulent Fluid Flow, Phys. Rev. Lett. 109, 134501 (2012).

[3] K. Gustavsson, J. Einarsson, and B. Mehlig, Tumbling of Small Axisymmetric Particles in Random and Turbulent Flows, Phys. Rev. Lett. 112, 014501 (2014).

[4] G. Voth and A. Soldati, Annu. Rev. Fluid Mech. (to be published).

[5] C. Marchioli, M. Fantoni, and A. Soldati, Orientation, distribution, and deposition of elongated, inertial fibers in turbulent channel flow, Phys. Fluids 22, 033301 (2010).

[6] N. R. Challabotla, L. Zhao, and H. Andersson, Orientation and rotation of inertial disk particles in wall turbulence, J. Fluid Mech. 766, R2 (2015).

[7] G. Voth, Disks aligned in a turbulent channel, J. Fluid Mech. 772, 1 (2015).

[8] P. G. Saffman, On the motion of small spheroidal particles in a viscous liquid, J. Fluid Mech. 1, 540 (1956).

[9] G. Subramanian and D. L. Koch, Inertial effects on fibre motion in simple shear flow, J. Fluid Mech. 535, 383 (2005).

[10] J. Einarsson, F. Candelier, F. Lundell, J. R. Angilella, and B. Mehlig, Rotation of a spheroid in a simple shear at small Reynolds number, Phys. Fluids 27, 063301 (2015).

[11] T. Rosén, J. Einarsson, A. Nordmark, C. K. Aidun, F. Lundell, and B. Mehlig, Numerical analysis of the angular motion of a neutrally buoyant spheroid in shear flow at small Reynolds numbers, Phys. Rev. E 92, 063022 (2015).

[12] H. Xu, A. Pumir, and E. Bodenschatz, The pirouette effect in turbulent flows, Nat. Phys. 7, 709 (2011).

[13] J. Jucha, H. Xu, A. Pumir, and E Bodenschatz, TimeReversal-Symmetry Breaking in Turbulence, Phys. Rev. Lett. 113, 054501 (2014).

[14] H. Xu, A. Pumir, G. Falkovich, E. Bodenschatz, M. Shats, H. Xia, N. Francois, and G. Boffetta, Flight-crash events in turbulence, Proc. Natl. Acad. Sci. U.S.A. 111, 7558 (2014).

[15] A. Pumir, H. Xu, E. Bodenschatz, and R. Grauer, SingleParticle Motion and Vortex Stretching in Three-Dimensional Turbulent Flows, Phys. Rev. Lett. 116, 124502 (2016).

[16] P. M. Lovalenti and J. F. Brady, The force on a bubble, drop or particle in arbitrary time-dependent motion at small Reynolds number, Phys. Fluids 5, 2104 (1993).

[17] J. Einarsson, F. Candelier, F. Lundell, J. R. Angilella, and B. Mehlig, Effect of weak fluid inertia upon Jeffery orbits, Phys. Rev. E 91, 041002(R) (2015).

[18] F. Candelier, J. Einarsson, F. Lundell, B. Mehlig, and J. R. Angilella, The role of inertia for the rotation of a nearly spherical particle in a general linear flow, Phys. Rev. E 91, 053023 (2015); Erratum, Phys. Rev. E92, 059901(E) (2015).

[19] S. Kim and S. J. Karrila, Microhydrodynamics: Principles and Selected Applications (Butterworth-Heinemann, Boston, 1991). 
[20] See Supplemental Material at http://link.aps.org/ supplemental/10.1103/PhysRevLett.117.204501 for details of the calculation, which includes Ref. [21].

[21] J. Happel and H. Brenner, Low Reynolds Number Hydrodynamics (Kluwer Academic, Dordrecht, 1983).

[22] M. R. Maxey, The gravitational settling of aerosol particles in homogeneous turbulence and random flow fields, J. Fluid Mech. 174, 441 (1987).

[23] K. Gustavson and B. Mehlig, Statistical models for spatial patterns of heavy particles in turbulence, Adv. Phys. 65, 1 (2016).

[24] C. J. Lin, J. H. Peery, and W. R. Schowalter, Simple shear flow around a rigid sphere: Inertial effects and suspension rheology, J. Fluid Mech. 44, 1 (1970).

[25] J. Meibohm, F. Candelier, T. Rosen, J. Einarsson, F. Lundell, and B. Mehlig (to be published).

[26] M. Guala, B. Lüthi, A. Liberzon, A. Tsinober, and W. Kinzelbach, On the evolution of material lines and vorticity in homogeneous turbulence, J. Fluid Mech. 533, 339 (2005).

[27] P. L. Johnson and C. Meneveau, Large-deviation statistics of vorticity stretching in isotropic turbulence, Phys. Rev. E 93, 033118 (2016).

[28] Y. Li, E. Perlman, M. Wan, Y. Yang, C. Meneveau, R. Burns, S. Chen, A. Szalay, and G. Eyink, A public turbulence database cluster and applications to study Lagrangian evolution of velocity increments in turbulence, J. Turbul. 9, N31 (2008).

[29] H. Yu, K. Kanov, E. Perlman, J. Graham, E. Frederix, R. Burns, A. Szalay, G. Eyink, and C. Meneveau, Studying Lagrangian dynamics of turbulence using on-demand fluid particle tracking in a public turbulence database, J. Turbul. 13, N12 (2012).

[30] B. Lüthi, A. Tsinober, and W. Kinzelbach, Lagrangian measurement of vorticity dynamics in turbulent flow, J. Fluid Mech. 528, 87 (2005).

[31] J. Schumacher, J. D. Scheel, D. Krasnov, D. A. Donzis, V. Yakhot, and K. R. Sreenivasan, Small-scale universality in fluid turbulence, Proc. Natl. Acad. Sci. U.S.A. 111, 10961 (2014).

[32] M. Byron, J. Einarsson, K. Gustavsson, G. Voth, B. Mehlig, and E. Variano, Shape-dependence of particle rotation in isotropic turbulence, Phys. Fluids 27, 035101 (2015).

[33] R. Gatignol, The Faxén formulae for a rigid particle in an unsteady non-uniform Stokes flow, J. Méc. Théor. Appl. 1, 143 (1983).

[34] M. R. Maxey and J. J. Riley, Equation of motion for a small rigid sphere in a nonuniform flow, Phys. Fluids 26, 883 (1983).
[35] H. Traugott and A. Liberzon, Experimental study of forces on freely moving spherical particles during resuspension into turbulent flow, Int. J. Multiphase Flow 88, 167 (2017).

[36] E. Variano (private communication).

[37] G. G. Marcus, S. Parsa, S. Kramel, R. Ni, and G. A. Voth, Measurements of the solid-body rotation of anisotropic particles in 3d turbulence, New J. Phys. 16, 102001 (2014).

[38] H. Homann and J. Bec, Finite-size effects in the dynamics of neutrally buoyant particles in turbulent flow, J. Fluid Mech. 651, 81 (2010).

[39] W. Fornari, F. Picano, G. Sardina, and L. Brandt, Reduced particle settling speed in turbulence, arXiv:1608.08549.

[40] K. Gustavsson, S. Vajedi, and B. Mehlig, Clustering of Particles Falling in a Turbulent Flow, Phys. Rev. Lett. 112, 214501 (2014).

[41] P. J. Ireland, A. D. Bragg, and L. R. Collins, The effect of Reynolds number on inertial particle dynamics in isotropic turbulence. Part 2. Simulations with gravitational effects, J. Fluid Mech. 796, 659 (2016).

[42] V. Mathai, E. Calzavarini, J. Brons, C. Sun, and D. Lohse, Microbubbles and Microparticles Are Not Faithful Tracers of Turbulent Acceleration, Phys. Rev. Lett. 117, 024501 (2016).

[43] H. Parishani, O. Ayala, B. Rosa, L.-P. Wang, and W. W. Grabowski, Effects of gravity on the acceleration and pair statistics of inertial particles in homogeneous isotropic turbulence, Phys. Fluids 27, 033304 (2015).

[44] H. R. Pruppacher and J. D. Klett, Microphysics of Clouds and Precipitation, 2nd ed. (Kluwer Academic, Dordrecht, 1997).

[45] R. Zimmermann, Y. Gasteuil, M. Bourgoin, R. Volk, A. Pumir, and J.-F. Pinton, Rotational Intermittency and Turbulence Induced Lift Experienced by Large Particles in a Turbulent Flow, Phys. Rev. Lett. 106, 154501 (2011).

[46] P. G. Saffman, The lift on a small sphere in a slow shear flow, J. Fluid Mech. 22, 385 (1965).

[47] S. Parsa and G. A. Voth, Inertial Range Scaling in Rotations of Long Rods in Turbulence, Phys. Rev. Lett. 112, 024501 (2014).

[48] Varghese Mathai, Vivek N. Prakash, Jon Brons, Chao Sun, and Detlef Lohse, Wake-Driven Dynamics of Finite-Sized Buoyant Spheres in Turbulence, Phys. Rev. Lett. 115, 124501 (2015).

[49] H. A. Stone, J. F. Brady, and P. M. Lovalenti, Inertial effects on the rheology of suspensions and on the motion of individual particle, preprint. 